\title{
Métodos iterativos eficientes para a Pseudo-Inversa com aplicações na construção de soluções aproximadas para sistemas lineares de grande porte
}

\section{Everton Boos ${ }^{1}$}

Departamento de Matemática, Centro de Ciências Físicas e Matemáticas, UFSC, Florianópolis, $\mathrm{SC}$

\section{Fermín S. V. Bazán²}

Departamento de Matemática, Centro de Ciências Físicas e Matemáticas, UFSC, Florianópolis, SC

\begin{abstract}
Resumo. Neste trabalho apresentamos métodos iterativos para o cálculo da matriz pseudoinversa, juntamente com resultados pertinentes sobre convergência. Além dos aspectos teóricos, são apresentadas implementações práticas no ambiente Matlab. Visto que o cálculo explícito da pseudo-inversa não é de interesse quando a matriz é de grande porte, são apresentadas versões vetoriais dos métodos baseadas em produtos matriz vetor, que convergem à solução do problema de mínimos quadrados.
\end{abstract}

Palavras-chave. Pseudo-inversa, Métodos iterativos, Sistemas lineares, Problemas de mínimos quadrados.

\section{Introdução}

O problema de resolver sistemas de equações lineares é assunto recorrente em diversas áreas das ciências aplicadas. Em geral, trata-se de resolver problemas da forma

$$
A x=b, \quad \text { ou } \quad \min \|A x-b\|_{2},
$$

com $A \in \mathbb{C}^{m \times n}, b \in \mathbb{C}^{m}$, e $x \in \mathbb{C}^{n}$ (vetor de incógnitas). Quando o sistema admite solução única, ela é dada por $x=A^{-1} b$ ou por $A^{+} b$ em que $A^{+}$é a matriz pseudo-inversa de $A$ [2]. É sabido que computar a matriz inversa não é recomendável devido ao alto custo computacional, portanto, o cálculo do produto $A^{-1} b$ pode ser inviável. A mesma observação é válida para o caso do produto $A^{+} b$.

Para contornar as dificuldades apontadas acima, partimos para métodos iterativos para aproximar a pseudo-inversa, mas tendo em mente que, ao invés de aproximarmos a

\footnotetext{
${ }^{1}$ everton_boos@grad.ufsc.br

${ }^{2}$ fermin.bazan@ufsc.br
} 
própria pseudo-inversa, o principal objetivo é calcular aproximações do efeito dela quando aplicada ao vetor $b$.

Em termos matemáticos, numa primeira etapa a ideia é gerar uma sequência $\left\{X_{k}\right\}_{k \in \mathbb{N}}$ de matrizes de modo que $X_{k} \longrightarrow A^{+}$, quando $k \longrightarrow \infty$. Tendo garantida a convergência de $X_{k} \longrightarrow A^{+}$, a ideia da segunda etapa é construir uma sequência de vetores $x^{(k)} \in \mathbb{C}^{n}$, baseada na sequência $X_{k}$, e analisar sob quais condições, $x^{(k)} \longrightarrow A^{+} b$.

\section{Os métodos iterativos}

Em geral, utilizamos uma aproximação inicial $X_{0}=\beta A^{*}$, para $\beta$ satisfazendo certas condições, e uma sequência de matrizes $\left\{X_{k}\right\}_{k \in \mathbb{N}}$ definidas na forma

$$
X_{k+1}=X_{k}+C_{k} T_{k}
$$

com $C_{k}$ e $T_{k}$ escolhidas de diferentes formas. Esta parte do estudo está baseada na referência [1]. Basicamente, se forem feitas escolhas corretas para as matrizes $C_{k}$ e $T_{k}$, prova-se que $X_{k} \longrightarrow A^{+}$, quando $k \longrightarrow \infty$. Obviamente, do ponto de vista computacional, realizam-se apenas uma certa quantidade de iterações até um critério de parada ser satisfeito. Notamos que, a partir da aproximação $X_{k}$ obtida, uma solução aproximada para o sistema linear ou para o problema de mínimos quadrados é obtida imediatamente através do produto $X_{k} b$. A outra alternativa é gerar a sequência de vetores

$$
x^{(k)}=X_{k} b \in \mathbb{C}^{n},
$$

interrompendo o processo através de um critério de parada apropriado. Se $A$ é de grande porte, é evidente que é mais econômico usar a sequência vetorial do que a sequência matricial.

Apresentamos resultados numéricos mostrando que os métodos iterativos apresentam convergência rápida à pseudo-inversa. Os métodos baseados em sequências vetoriais para aproximar $A^{+} b$ estão em fase de implementação e análise.

\section{Referências}

[1] A. Ben-Israel and T. N. E. Greville, Generalized inverses: theory and applications, Springer, 2nd edition, (2003).

[2] C. D. Meyer, Matrix analysis and applied linear algebra, SIAM, (2000). 